NEURODEGENERATIVE DISEASE

\section{Expanding \\ neurodegeneration modelling}

Frontotemporal dementia (FTD) and amyotrophic lateral sclerosis (ALS) are diseases that have overlapping cognitive and behavioural symptoms. The most common genetic cause known for both disorders is a G4C2 repeat expansion on chromosome 9 open reading frame 72 (C9ORF72), and thus FTD and ALS with this genetic abnormality are collectively referred to as c9FTD/ALS. The currently poor understanding of how the C9ORF72 expansion results in c9FTD/ALS has much to do with the lack of models that reproduce the key aspects of disease phenotypes. In this study, the authors successfully developed a mouse model of c9FTD/ALS that recapitulates many of the cellular, behavioural and clinical phenotypes observed in humans.

In humans, the expanded C9ORF72 can be bidirectionally transcribed, and C9ORF72 transcripts form intranuclear foci. Moreover, these transcripts lack an ATG start codon and so are prone to repeat-associated non-ATG (RAN) translation, which results in the production of c9RAN proteins that also form inclusions in affected cells.

The authors used an adenoassociated viral vector to induce the expression of either $2 \mathrm{G} 4 \mathrm{C} 2$ ((G4C2)2; controls) or (G4C2)66 (disease model) repeats in the CNS at postnatal day 0 . The pathological changes in the mice were analysed 6 months later. Using fluorescence in situ hybridization to detect bidirectionally transcribed RNA from the G4C2 repeat, the authors found that nuclear foci were widespread throughout the CNS of (G4C2)66 mice, including in the cortex, hippocampus, cerebellum and thalamus, but not in control animals. Nuclear and cytoplasmic c9RAN protein inclusions were also detected throughout the CNS in only (G4C2)66 mice. Neuronal loss was noted in the cortex and cerebellum but not in the hippocampus or thalamus.

Another pathological hallmark of c9FTD/ALS is the presence of phosphorylated TAR DNA-binding protein 43 (pTDP43). (G4C2)66 mice exhibited nuclear and sometimes cytoplasmic pTDP43 inclusions in $7-8 \%$ of cortical and hippocampal cells. Importantly, these data indicate that the G4C2 repeat expansion is upstream of TDP43 pathology in c9FTD/ALS.

Next, the 6-month-old mutant mice were subjected to a range of tests to analyse behaviours associated with c9FTD/ALS. In the open-field assay, (G4C2) 66 mice spent less time on the centre of the open arena than did controls, which indicates

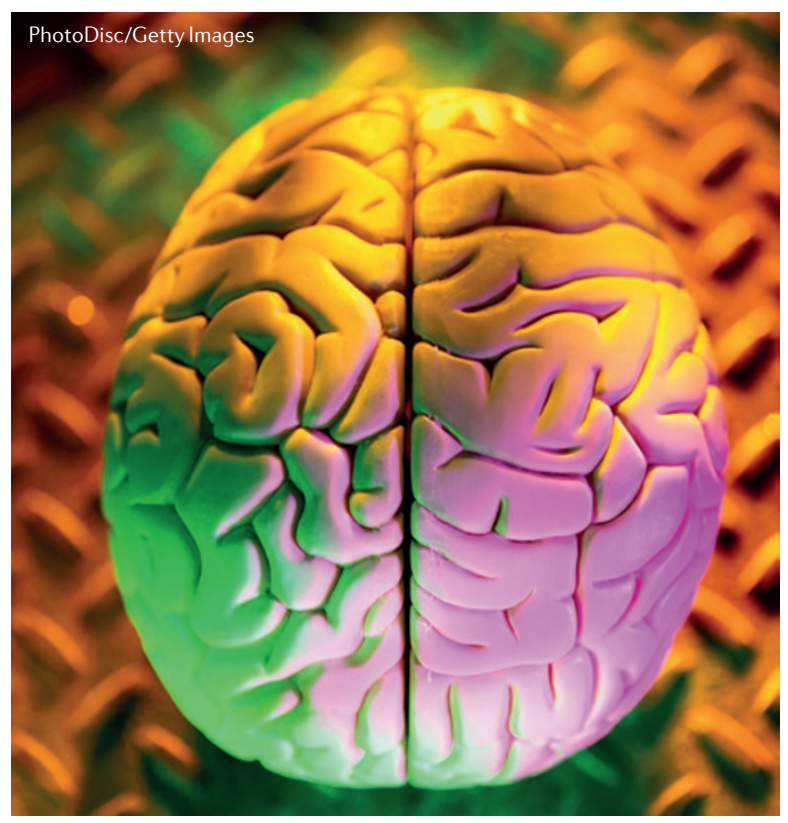

increased anxiety-like behaviour. In other behavioural tests, (G4C2)66 mice showed motor impairments and reduced social interaction compared with controls.

Overall, these findings indicate that the (G4C2)66 mouse could be a useful model of c9FTD/ALS that recapitulates key pathophysiological and behavioural phenotypes. The model could also be used to develop potential therapeutic approaches that would not only mitigate G4C2 repeat expansion-associated pathologies but also the underlying downstream pathology: namely, that of TDP43.

Sian Lewis

ORIGINAL RESEARCH PAPER Chew, J. et al. C9ORF72 repeat expansions in mice cause TDP-43 pathology, neuronal loss, and behavioral deficits. Science http://dx.doi.org/10.1126/science.aaa9344 (2015) the (G4C2)66

mouse could

be a useful

model of

c9FTD/

ALS that recapitulates key pathophysiological and behavioural phenotypes 